\title{
THE REDSHIFTS OF EXTRAGALACTIC OBJECTS
}

\author{
WALLACE L. W. SARGENT
}

Hale Observatories, California Institute of Technology, Carnegie Institution of Washington, U.S.A.

\begin{abstract}
We review the evidence for a general shift-distance law for galaxies. This is shown directly by a redshift-distance diagram recently prepared by Sandage and Tammann for Sc I galaxies. The distances are measured from $\mathrm{H}$ II region diameters and from the diameters of the Sc I galaxies themselves for the more distant systems. We also show the redshift-apparent magnitude diagrams for several objects which are identifiable over a large range in distance and which prove to have a small range in absolute magnitude: these are supernovae of type I (Kowal and Sargent, 1973, unpublished), brightest cluster galaxies (Sandage, 1972a), and radio galaxies (excluding N-type galaxies) (Sandage, 1972b). Plots of redshift versus angular size for brightest cluster galaxies (Sandage, 1972c) also have the correct slope for this to be a distance effect. In summary, the tightness of the correlations for the various diagrams listed implies that there is a very close correlation between distance and redshift for galaxies out to at least $z=\Delta \lambda / \lambda \sim 0.2$ and that there is no evidence for redshift anomalies among the objects in these diagrams.

The interpretation of the cosmological redshift as a Doppler shift due to the expansion of the Universe is established only by indirect arguments (Peebles, 1971). A repetition of the test proposed by Hubble and Tolman (1935), which involves a correlation of surface brightness and redshift, would be important in this connection.

We discuss possible examples of redshift anomalies in galaxies. These include (a) compact groups of galaxies - Stephan's Quintet, VV172, Zwicky's triple system and Seyfert's Sextet - which each have one highly discrepant member; (b) Tifft's (1972a, $1972 \mathrm{~b}$, see also p. 243) work on band structure in the redshift-magnitude diagrams for clusters of galaxies; (c) the systematic difference in redshift between spirals and ellipticals in the Virgo cluster (Holmberg, 1961 ; Tammann, 1972); (d) Arp's work on galaxies with apparent connections to high redshift companions - NGC 7603, NGC 772; (e) Arp's work on the redshifts of dwarf companions to large spiral galaxies (Arp, 1971; Lewis, 1971). We find that there is no compelling reason to believe that redshift anomalies are responsible for any of the phenomena exhibited by these systems. There have been no proper statistical studies which would eliminate the possibility of chance projections of background or foreground galaxies being responsible for some anomalies. In particular Lynds' (1972) work shows convincingly that the discrepant object, NGC 7320, in Stephan's Quintet is due to such a chance superposition. In the case of Tifft's work the fact that the band structure observed for the Virgo cluster is nearly orthogonal to that claimed for the Coma cluster makes his interpretations highly questionable.

We next consider the redshifts of N-type galaxies. The conventional view is that the $\mathrm{N}$-galaxies are 'mini-Quasars' although it is also possible that the $\mathrm{N}$-galaxies and
\end{abstract}


quasars form a sequence of increasing non-cosmological contribution to the redshift. Sandage (1973) showed that it is possible to decompose the light of $\mathrm{N}$-galaxies into a non-thermal component and a component with colours similar to those of a normal galaxy. Moreover, in a plot of the redshift of the $\mathrm{N}$-galaxy versus the magnitude of the 'galaxy' component, the points fall on the line defined by normal radio ellipticals. Thus there is no evidence for a non-cosmological contribution to the redshifts of $\mathrm{N}$-galaxies out to $z \sim 0.2$. The same result is found for some individual $\mathrm{N}$-type systems (3C 371, III Zw 2, B 264, RN 8) which are in clusters or which have normal companion galaxies with the same redshift.

Finally we discuss the present evidence which bears on the quasar distances. The evidence points strongly, but not conclusively, to cosmological distances for these objects. The work by Gunn and his associates on quasars in clusters with the same redshift ( 5 cases out of 7 so far investigated) and Kristian's (1973) evidence for galaxies around quasars are particularly compelling. At the same time the features which have contradicted the cosmological viewpoint have tended to disappear with the passage of time. Among these we may cite (a) the distribution of redshifts over the sky is no longer thought to be highly anomalous (Wills, 1972); (b) according to Burbidge (1973) there is no longer any reason to believe in anomalous peaks or in periodicities in the distribution of quasar redshifts; the remaining anomalies are associated with peculiar emission-line objects, such as radio galaxies, that are not quasars; (c) the anomalous proximity of four 3CR quasars to bright galaxies in the Reference Catalogue has not been found in other samples of quasars and galaxies; (d) the chain of quasars near NGC 520 pointed out by Arp (1970) looks much less impressive when all quasars in that region of the sky are considered.

Just before the Symposium, Wampler et al. (1973) discovered that the radio source $4 \mathrm{C} 11.50$ is a double quasar $4.8^{\prime \prime}$ apart. The components are $17^{\mathrm{m}}$ and $19^{\mathrm{m}}$ and have redshifts $z=0.435$ and $z=1.901$, respectively. At first it appeared that there was an absorption line corresponding to $\mathrm{Mg}$ II $\lambda 2800$ in absorption in the high redshift object at a wavelength corresponding to the low redshift. Even more surprising there seemed to be weak emission features corresponding to the high redshift in the low redshift object. Later observations did not reveal these emission features. However, the close proximity of the two objects has a low probability on the cosmological hypothesis where it would have to be accidental. A large number of such pairs would be hard to account for in conventional terms.

\section{References}

Arp, H. C.: 1970, Astron. J. 75, 1.

Arp, H. C.: 1971, Nature Phys. Sci. 231, 103.

Burbidge, G. R.: 1973, Mitt. Astron. Gesellsch., Nr. 34, p. 19.

Holmberg, E.: 1961, Astron. J. 66, 620.

Hubble, E. and Tolman, R. C.: 1935, Astrophys. J. 82, 302.

Kristian, J.: 1973, Astrophys. J. Letters 179, L61.

Lewis, B. M.: 1971, Nature Phys. Sci. 231, 13. 
Lynds, C. R.: 1972, in D. S. Evans (ed.), 'External Galaxies and Quasi-Stellar Objects', IAU Symp. 44, 376.

Peebles, P. J. E.: 1971, Comments Astrophys. Space Sci. 3, 173.

Sandage, A. R.: 1972a, Astrophys. J. 178, 1.

Sandage, A. R.: 1972b, Astrophys. J. 178, 25.

Sandage, A. R.: 1972c, Astrophys. J. 173, 485.

Sandage, A. R.: 1973, Astrophys. J. 180, 687.

Tammann, G.: 1972, Astron. Astrophys. 21, 355.

Tifft, W. G.: 1972a, in D. S. Evans (ed.), 'External Galaxies and Quasi-Stellar Objerts', IAU Symp. 44, 367.

Tifft, W. G.: 1972b, Astrophys. J. 175, 613.

Wampler, E. J., Robinson, E. L., Baldwin, J. A., Hazard, C., and Jauncey, D. L.: 1973, Nature 246, 203.

Wills, D.: 1972, Nature Phys. Sci. 238, 70.

\section{DISCUSSION}

G. Burbidge: We (Burbidge, G. R. and O'Dell, S. L.: 1973, Astrophys. J. 183, 759) have recently re-done the work by Bahcall and Hills (Astrophys. J. 179, 699, 1973) on the redshift-magnitude relationship for the brightest QSOs and we get a slope of about 4 rather than their value of 5 . The slope turns out to depend critically on the presence of $3 \mathrm{C} 273$. If it is left out, the slope is only two, which shows - that the slope is not well-determined at all.

Sargent: - that 3C 273 ought to be put in!

G. Burbidge: Although the recent work on the peaks at $z-0.06$ and 1.95 in the number vs redshift distribution suggests that they are not absolutely established on a statistical basis, I still believe that there's something significant about them.

Bolton: Two comments:

(i) regarding Kristian's evidence on underlying galaxies. I looked at these objects on the Sky Survey prints and in all but one case I believe the objects should have been called galaxies in the first place.

(ii) On the close QSOs, Peterson and I have found five cases in the southern sky of pairs of QSOs within about $30^{\prime \prime}$ of each other. One of each pair is identified with a radio source.

Sargent: In reply to those two points:

(i) If you are referring to the objects near the line representing the Hubble relationship, then what you say is to be expected (on the cosmological interpretation of redshifts). The important fact, which must be explained by those who hold the non-cosmological interpretation of redshifts, is why those objects away from the line do not show evidence of a 'fuzz' around them.

(ii) Wampler estimates that there are about $10^{6}$ QSOs in the sky with magnitudes brighter than 19. The probability is therefore about $10^{-4}$ of finding one of these lying within $5^{\prime \prime}$ of a particular QSO and; correspondingly, one expects over the whole sky about 100 pairs within $5^{\prime \prime}$ of each other. If the QSOs selected are bright ones then, of course, the expected number of pairs goes down.

Heidmann: Arp's statistics on the redshift differences between main and companion galaxies have recently been redone by Bottinelli and Gouguenheim (Astron. Astrophys. 26, 85, 1973) using all of the 50 groups in de Vaucouleurs' Chapter 17 (Stars and Stellar Systems, vol. 9). Arp's result is plainly confirmed, the difference being $90 \mathrm{~km} \mathrm{~s}^{-1}$ with a $30 \mathrm{~km} \mathrm{~s}^{-1}$ mean error; so the zero value is excluded at the $3 \sigma$ level.

Sargent: My disagreement is not about the ability of astronomers to subtract one number from another, but whether the numbers are right. The systematic effects must be looked at very carefully.

Heidmann: These have been investigated by Bottinelli and Gouguenheim and I refer you to their paper.

Abell: Calculation of the probability of finding a particular configuration of objects in the sky is very tricky. When a particular pattern is already observed, one cannot say that it is as unlikely to have happened by chance as the probability of it having occurred at random if that pattern were described in advance of its discovery. Suppose, for example, one numbered 1000 pieces of paper as 1 to 1000 , and put them into a box; then suppose one paper is drawn at random and is found to be, say, number 633 . The chance that no. 633 would be drawn, if named in advance, is 0.001 , but that 
is the same probability as any other number, and one had to be drawn.

In the case of Wampler's discovery of a $19^{\mathrm{m}}$ quasar $5^{\prime \prime}$ from a $17^{\mathrm{m}}$ quasar, Sargent has reminded us that one would expect 100 examples of two quasars within 5 " of each other. One such pair happens to have been discovered to date.

G. Burbidge: There is also Stockton's pair (Nature Phys. Sci. 238, 37, 1972).

Arp: The statement by Sargent about the line of QSRs from NGC 520 should be corrected. I plotted only faint $\left(V<17^{\mathrm{m}}\right)$ QSRs; he plotted also PHL - radio-quiet objects of all apparent magnitudes, which are not a complete sample and from which no conclusions can be drawn.

If you restrict yourself to a homogeneously selected sample but over a larger area than I originally considered, then the two QSRs on the other side of the chain of four QSRs appear significant. I never discussed them because they were further out and one or two others fell off the chain. But of the two QSRs in a line opposite the original chain, one has a redshift almost exactly equal to a quasar in the original chain. Sargent's diagram makes it look as though there is a cluster of QSOs around NGC 520 I never mentioned this situation because of the incompleteness of this sample - but if Sargent believes the sample is complete he should explain the apparent clustering around NGC 520.

Sargent: Do you wish me to reply to that, or can we go for a beer? 